\title{
Review of: "Assessing acceptance of augmented reality in nursing education"
}

\author{
Kim Geok Chan ${ }^{1}$ \\ 1 Universiti Malaysia Sarawak
}

Potential competing interests: The author(s) declared that no potential competing interests exist.

In this study, the use of the Google Play Store downloaded application such as the AsthiAR and SnapLearn on human anatomy in students' mobile phone was found to be beneficial to nursing students. It is one of the mobile apps which is flexible, accessible; actively involved students.

The study reports finding of general user acceptance of augmented reality in nursing education which support its use to motivate students' self-learning. It was perceived to improve the students' performance academically. It was reported to present a holistic way of teaching for certain courses and creates an alternative to conventional textbooks. It further claims that nursing students can improve their clinical nursing skills in addition to learning theoretical knowledge and techniques using the AR technology. Issues which could have been addressed for this study for a more convincing results: these include: Study was done among nursing students base on voluntary basis, no estimation of how much duration of use, not mandatory for its use. The effects of AR technology on academic performance was not measured, and compared with control group. One other related issue as noticed was, the sample of nursing students comprised of undergrad students and graduate students: there would be differences in the years of study and experience, hence, variation in their responses.

Without doubt, there will be benefits of using of existing AR mobile apps for self-learning, to enhance, supplement the learning of nursing students, in particular, basic medical science related courses in the early years of their studies. This is so, especially during the current covid pandemic when classes are conducted online instead of face to face. The provision of clinical learning opportunities for nursing students to gain experiences of clinical nursing at patients' bedside is definitely an essential of nursing education. 